\title{
The Complexation of Silver (I) ion with 18-Crown-6 Ether in Water and Some Polar Aprotic Solvents
}

\author{
Denis A. Feofanov, Maxim Yu. Penin, \\ Oleg A. Epov and Pavel V. Fabinskii* \\ Reshetnev Siberian State University \\ of Science and Technology \\ 82 Mira, Krasnoyarsk, 660049, Russia
}

Received 16.04.2019, received in revised form 19.04.2019, accepted 26.08.2019

\begin{abstract}
The process of complexation of silver (I) ions with 18-crown-6 ether in some polar water-aproton solvents at a constant temperature of $308 K$ was studied by direct potentiometric titration. The stability constants of the resulting complex are determined depending on the molar fraction from 0.2 to 0.8 dimethylsulfoxide and dimethylformamide in the composition of the mixed solvent. It is shown that, despite the same nature of the co-solvents, they have the opposite effect on the stability constant of the complex due to the instability of DMF in an acidic medium and when heated. It is noted that the studied reaction is exothermic and the temperature increase reduces the stability of the studied complexes.
\end{abstract}

Keywords: stability constant, silver (I) ion, 18-crown-6 ether, titration potentiometry, mixed aqueousorganic solvent.

Citation: Feofanov D.A., Penin M.Yu., Epov O.A., Fabinskii P.V. The complexation of silver (I) ion with 18-crown-6 ether in water and some polar aprotic solvents, J. Sib. Fed. Univ. Chem., 2019, 12(3), 374-381. DOI: 10.17516/1998-2836-0134.

(C) Siberian Federal University. All rights reserved

* Corresponding author E-mail address:knx@sibsau.ru 


\title{
Комплексообразование ионов серебра (I) \\ с 18-краун-6 эфиром в некоторых полярных \\ водно-апротонных растворителях
}

\author{
Д.А. Феофанов, М.Ю. Пенин, \\ О.А. Эпов, П.В. Фабинский \\ Сибирский государственный университет науки \\ и технологий имени академика М.Ф. Решетнева \\ Россия, 660049, Красноярск, пр. Мира, 82
}

\begin{abstract}
Методом прямого потенциометрического титрования изучен процесс комплексообразования ионов серебра (I) с 18-краун-6 эфиром в среде некоторых полярных водно-апротонных растворителей при постоянной температуре $308 \%$ Константы устойчивости образующегося комплекса определены при различных мольных долях диметилсульфоксида и диметилформамида от 0,2 до 0,8 в составе смеманного растворителя. Показано, что, несмотря на одинаковую природу сорастворителей, они оказывают прямо противоположное действие на константу устойчивости комплекса вследствие неустойчивости ДМФА в кислой среде и при нагревании. Отмечено, что исследуемая реакция комплексообразования является экзотермичной, а повышение температуры уменьшает устойчивость исследуемых комплексов.
\end{abstract}

Ключевые слова: константа устойчивости, ион серебра (I), 18-краун-6 эфир, потенциометрия титрования, смешанный водно-органический растворитель.

\section{Введение}

Несмотря на наличие большого числа экспериментальных данных по изучению устойчивости комплексных соединений в разных растворителях, включая смешанные, установленные закономерности во взаимосвязи констант устойчивости образующихся комплексов с природой лигандов и средой носят лишь частный характер [1]. Для выявления более общих закономерностей процессов комплексообразования и интерпретации полученных экспериментальных данных необходимо получение значений констант устойчивости при варьировании температуры, ионной силы и замены среды.

Краун-эфиры - вещества с широким спектром применения, которые способны образовывать комплексы с высокой степенью селективности по отношению к катионам $[2,3]$. Данная избирательность напрямую зависит как от размеров молекулярной полости эфира, так и от радиуса центрального иона комплексообразователя, и в зависимости от этих факторов могут быть использованы разного вида краун-эфиры $[4,5]$. По отношению к ионам серебра (I) наиболее селективен 18-краун-6 эфир. Процесс комплексообразования этой системы изучен при температуре 298,15 К как в индивидуальных растворителях (метанол [6, 7], этанол [8, 9], N-метил2-пирролидон [10], ДМСО [11, 12], ДМФА [13, 14]), так и в смешанных [15-17].

$$
-375-
$$


Природа растворителя - один из факторов управления процессом комплексообразования в растворах. В качестве растворителей, где исследовался процесс комплексообразования ионов серебра (I) с 18-краун-6 эфиром (1,4,7,10,13,16-гексаоксациклооктадекан) при температуре 308К, были выбраны смеси воды и двух апротонных растворителей: полярного N,Nдиметилформамида (ДМФА) и биполярного диметилсульфоксида (ДМСО). Полярность растворителей в сочетании со способностью краун-эфиров образовывать комплексы позволила ввести неорганический компонент в среду органического растворителя [18, 19].

\section{Экспериментальная часть}

Исследование процесса комплексообразования проводили методом прямого потенциометрического титрования раствора нитрата серебра (I) раствором 18-кран-6 эфира (18К6) при постоянной температуре 308 К. Прямая потенциометрия позволяет упростить расчет ЭДС, поскольку отсутствует диффузионный потенциал, появляющийся при переносе заряда по электролитическому мостику. В качестве индикаторного электрода использовали металлический серебряный электрод. Для сравнения брали лабораторный стеклянный электрод ЭСЛ-45-11. Электроды были подготовлены и откалиброваны по воспроизведению электродной функции к обратимому иону по стандартным методикам [20]. Следует отметить, что другие виды электродов сравнения применять достаточно проблематично, поскольку попадание в раствор посторонних ионов металлов создает конкурирующую реакцию с лигандом и изменяет ионную силу раствора, что отражается на ЭДС ячейки.

Титруемые растворы, помимо основных участников процесса комплексообразования, дополняли хлорной кислотой с концентрацией 0,45 моль/л. Введение ее в систему позволяет обеспечить работу стеклянного электрода, а также подавить гидролиз соли серебра (I) в процессе измерения $[20,21]$. Перемешивание раствора осуществлялось методом барботирования очищенного газообразного азота через титруемый раствор. Постоянство температуры достигалось погружением измерительной ячейки в жидкостный термостат типа KRIOVIST-5 на весь период эксперимента. Контролировали температуру термометром сопротивления, показания которого имеют погрешность $\pm 0,2{ }^{\circ} \mathrm{C}$.

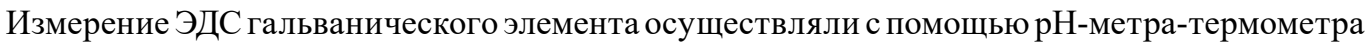
с высоким входным сопротивлением типа НИТРОН-рН с точностью \pm 1 мВ. Запись показаний ЭДС и температуры раствора проводили в режиме реального времени. Каждая точка титрования выдерживалась для термостатирования исследуемого раствора и достижения постоянного значения ЭДС измерительной ячейки. Схема электродной системы для измерения ЭДС имеет вид

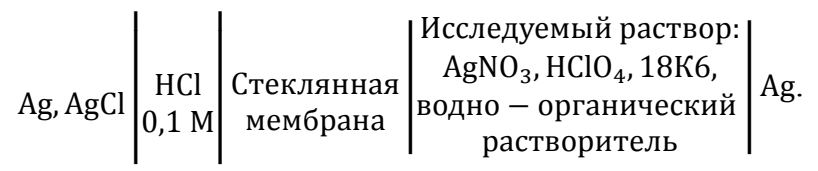

Для изучения влияния содержания ДМСО и ДМФА в составе смешанного водноорганического растворителя на константу устойчивости комплекса серебра (I) с краун-эфиром были приготовлены титруемые растворы с мольными долями апротонных сорастворителей, равными: $0.1,0.2,0.3,0.4,0.5,0.6,0.7$ и 0,8 . 


\section{Результаты и их обсуждение}

Реакции комплексообразования в смешанных водно-органических растворителях сложные и многоступенчатые. В системе $\mathrm{H}_{2} \mathrm{O}$ - ДМСО или $\mathrm{H}_{2} \mathrm{O}$-ДМФА можно выделить несколько основных процессов, протекающих в растворе:

$$
\begin{aligned}
& \mathrm{Ag}^{+}+2 \mathrm{H}_{2} \mathrm{O} \rightleftarrows\left[\mathrm{Ag}\left(\mathrm{H}_{2} \mathrm{O}\right)_{2}\right]^{+}, \\
& {\left[\mathrm{Ag}\left(\mathrm{H}_{2} \mathrm{O}\right)_{2}\right]^{+}+18 \mathrm{~K} 6 \rightleftarrows[\mathrm{Ag}(18 \mathrm{~K} 6)]^{+}+2 \mathrm{H}_{2} \mathrm{O},} \\
& {\left[\mathrm{Ag}\left(\mathrm{H}_{2} \mathrm{O}\right)_{2}\right]^{+}+\mathrm{n}\left(\mathrm{CH}_{3}\right)_{2} \mathrm{SO} \rightleftarrows\left[\mathrm{Ag}\left(\left(\mathrm{CH}_{3}\right)_{2} \mathrm{SO}\right)_{\mathrm{n}}\right]^{+}+2 \mathrm{H}_{2} \mathrm{O},} \\
& {\left[\mathrm{Ag}\left(\mathrm{H}_{2} \mathrm{O}\right)_{2}\right]^{+}+\mathrm{n}\left(\mathrm{CH}_{3}\right)_{2} \mathrm{NCOH} \rightleftarrows\left[\mathrm{Ag}\left(\left(\mathrm{CH}_{3}\right)_{2} \mathrm{NCOH}\right)_{\mathrm{n}}\right]^{+}+2 \mathrm{H}_{2} \mathrm{O} .}
\end{aligned}
$$

Процесс, отражаемый уравнением (1), наблюдается в водных растворах солей серебра (I), поскольку $\mathrm{Ag}^{+}$окружены гидратной оболочкой и существуют в виде аквакомплексов катионного типа [22]. При проведении потенциометрического титрования происходит смешение и в образующемся растворе протекают несколько конкурирующих процессов комплексообразования, связанных с заменой в аквакомплексе серебра (I) лигандов на 18-краун-6 эфир (2) или на молекулы одного из полярных апротонных сорастворителей из состава смешанного растворителя (3) либо (4). Следует отметить, что на примере данных систем подтверждается известное общее правило эффекта среды: чем сильнее полярность растворителя (сорастворителя в составе смешанного раствора), тем сильнее сказывается его влияние на комплексообразование [18].

Из нескольких возможных процессов, протекающих в исследуемой системе, объектом нашего изучения стала реакция образования комплекса серебра (I) с 18-краун-6 эфиром. Процесс образования коронатного комплекса (упрощенно и схематически) можно отобразить с помощью уравнения (5):

$$
18 \mathrm{~K} 6+\mathrm{Ag}^{+} \rightleftarrows[\operatorname{Ag}(18 \mathrm{~K} 6)]^{+} .
$$

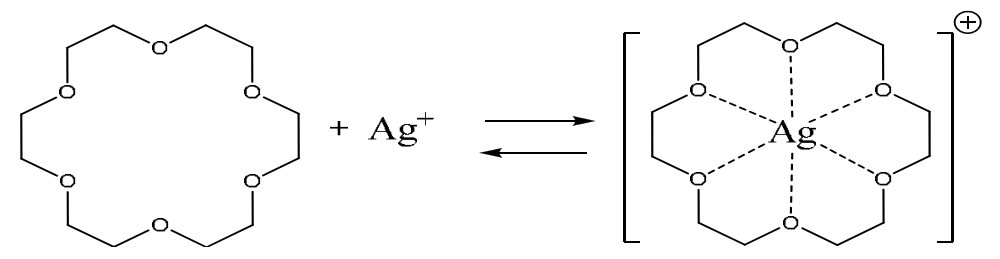

Выражение константы устойчивости (К) комплекса, образующегося по уравнению (5), имеет вид

$$
\mathrm{K}=\frac{\left[\mathrm{Ag}(18 \mathrm{~K} 6)^{+}\right]}{\left[\mathrm{Ag}^{+}\right][18 \mathrm{~K} 6]}
$$

Зависимости логарифма константы устойчивости комплекса серебра (I) с краун-эфиром от мольной доли органического компонента в составе смешанного растворителя представлены на рис. 1.

Константы устойчивости при 298 К для аналогичных систем, представленные на рис. 1, взяты из работы [23]. На начальном этапе и при незначительных добавках к воде ДМСО или ДМФА (до 0,2 м.д.) устойчивость исследуемого комплекса возрастает и значения логарифмов

$$
-377-
$$



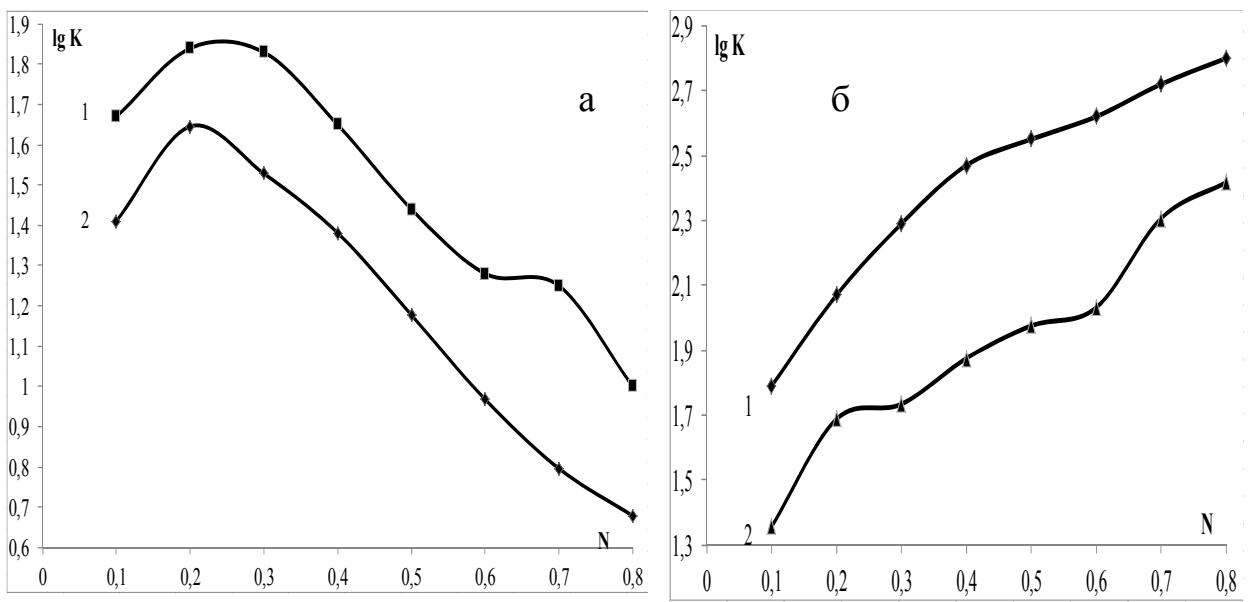

Рис. 1. Зависимости логарифма константы устойчивости комплекса серебра (I) с краун-эфиром от мольной доли ДМСО (а), ДМФА (б) при температуре 298 К (1) и 308 К (2)

Fig. 1. Dependences of the logarithm stability constant of the silver (I) complex with crown ether on the molar fraction of DMSO (a), DMF (б) at $298 \mathrm{~K}$ (1) and $308 \mathrm{~K}$ (2)

констант устойчивости практически одинаковы и не зависят от природы сорастворителя. Последующее увеличение содержание органического компонента (более 0,2 м.д.) в составе смешанного растворителя приводит к прямо противоположному ходу изменения кривых $\lg \mathrm{K}$.

Характер зависимости от состава смешанного растворителя констант устойчивости, полученных нами при температуре 308 К, и литературные данные [11-13], полученные авторами при стандартной температуре, практически не отличаются. Экстремальный характер изменения константы устойчивости в смесях воды и ДМСО авторы [11] объясняют динамикой двух сольватационных вкладов противоположной направленности при изменении состава смешанного растворителя.

Увеличение содержания ДМСО в составе смешанного растворителя приводит к уменьшению устойчивости комплексного иона (рис. $1 a$ ), что может быть объяснено значительным превалированием равновесия (3) по сравнению с равновесием (2). ДМСО относится к апротонным и биполярным растворителям, полярность его, оцененная в значениях диэлектрической проницаемости, почти на 10 единиц превосходит полярность ДМФА [18]. Ионы серебра (I), согласно литературным данным [24], имеют большое сродство к сере и образуют устойчивые и стабильные комплексы. Для амбидентантной молекулы ДМСО координация через кислородный атом является более распространенным вариантом, чем через атом серы, за исключением очень мягких кислот, таких как катионы платиновых металлов [25]. Поскольку ион серебра (I) относится к достаточно мягким акцепторам, то однозначный ответ на вопрос о координации в комплексе с ДМСО был получен при изучении кристаллической структуры $\left[\mathrm{Ag}\left(\left(\mathrm{CH}_{3}\right)_{2} \mathrm{SO}\right)_{2}\right] \mathrm{ClO}_{4}$ методом PCA [26]. Было установлено, что ион серебра (I) образует связи с молекулами ДМСО через атомы кислорода.

В системе вода - ДМФА (рис. 1б) с увеличением мольной доли органического сорастворителя в составе смешанного растворителя устойчивость комплексного иона $[\mathrm{Ag}(18 \mathrm{~K} 6)]^{+}$возрастает практически линейно $\left(\mathrm{R}^{2}=0,962\right)$. Это свидетельствует о том, что из конкурирующих в 
этой системе процессов, отражаемых уравнениями (2) и (4), более устойчив комплекс серебра с краун-эфиром, чем с ДМФА. Такой факт можно объяснить тем, что сольватирующее действие на ион серебра (I) ДМФА меньшее, чем у ДМСО, и связано не только с меньшей полярностью данного растворителя. Титруемые растворы содержали сильную хлорную кислоту, и процесс осуществлялся при нагревании (308 К), а известно [27], что ДМФА при данных условиях неустойчив, гидролизуется, поэтому выступать в качестве лиганда в процессе комплексообразования менее способен.

Сопоставляя изменения логарифма константы устойчивости комплексного иона $[\operatorname{Ag}(18 \mathrm{~K} 6)]^{+}$ в изученных системах смешанных растворителей (ДМСО и ДМФА), можно проследить, что при всех мольных долях органического компонента повышение температуры приводило к снижению устойчивости комплекса. Аналогичные изменения $\lg \mathrm{K}$ были прослежены и при изучении комплексообразования некоторых катионов щелечноземельных металлов с 18-краун-6 эфиром в водном растворе калориметрическим методом [28]. Анализ термодинамических характеристик комплексообразования выявил, что стандартная энергия Гиббса этого процесса в растворе практически полностью определяется изменением энтальпии. Исследуемые реакции являются экзотермическими, а величина $\Delta$ Н процесса комплексообразования линейно связана с температурой.

\section{Список литературы}

1. Абросимов В.К., Крестов А.Г., Альпер Г.А. Достижения и проблемы теории сольватации: структурно-термодинамические аспекты. М.: Наука, 1998. 246 с. [Abrosimov V.K., Cross A.G., Alper G.A. Achievements and problems of solvation theory: structural and thermodynamic aspects. Moscow, Nauka, 1998. 246 p. (In Russ.)].

2. Хираока М. Краун-соединения. Свойства и применение. М.: Мир, 1986. 363 с. [Hiraoka M. Crown Compounds. Their Characteristics and application. Moscow, Mir, 1986. 363 p. (In Russ.)].

3. Фегтле Ф. Химия комплексов «гость - хозяин». М.: Мир, 1988. 511 с. [Fegtle F. Chemistry of "guest - host" complexes. Moscow, Mir, 1988. 511 p. (In Russ.)]

4. Kuz'mina L.G., Demina L.I., Demin S.V., Zhilov V.I., Tsivadze A.Y., Shokurova N.A. Study of the Structure of Lithium Thiocyanate Complexes with Benzo-15-Crown-5 by X-ray Diffraction and IR Spectroscopy. Russian Journal of Inorganic Chemistry. 2018. Vol. 63(3), P. 357-360.

5. Kostikova G.V., Krasnova O.G., Demina L.I. Complaxation in solvent extraction systems of benzo-15-crown-5-chloroform substituted acetic acid. Russian Journal of Inorganic Chemistry 2018. Vol. 63(12), P. 1663-1666.

6. Lee S.S, Park S.O., Jung J.H. Conductance Study on the Characteristics of Solution Containing Crown Ethers and Univalent Cation Perchlorates. Bulletin of the Korean Chemical Society. 1990 Vol. 11(4), P. 276-281.

7. Buschmann H.-J. Stability constants and thermodynamic values for the formation of 1:1 -and 2:1 complexes of crown ethers with alkali and alkaline-earth ions in methanol. Chemische Berichte 1985. Vol. 118(7), P. 2746-2756.

8. Buschmann H.-J., Schollmeyer E. The complexation reaction of 18 -crown-6 with $\mathrm{Ag}^{+}$in different solvents studied by potentiometric and calorimetric methods. Inorganica Chimica Acta 2000. Vol. 298(1), P. 120-122.

$$
-379-
$$


9. Pouretedal H.R., Shamsipur M. Competitive Potentiometric Study of Complexation of Some Organoammonium Ions with Selected Crown Ethers in Ethanol Solution Using $\mathrm{Ag}^{+}$Ion as a Probe. Journal of Chemical \& Engineering Data 1998. Vol. 43(5), P. 742-744.

10. Kalidas C., Balaji S. Stabibty constants of silver (I) perchlorate complex with macrocyclic ligands in methanol + N-methyl-2-pyrrolidinone mixtures. Indian Journal of Chemistry 1999. Vol. 38A(6), P. 618-620.

11. Усачева Т.Р., Леденков С.Ф., Шарнин В.А. Влияние состава растворителя вода-ДМСО на термодинамику реакции образования [Ag18K6]. Координационная химия. 2001. Т. 27(3). C. 222-226. [Usacheva T.R., Ledenkov S.F., Sharnin V.A. The influence of the solvent composition of water-DMSO on the thermodynamics of the reaction of formation [Ag18K6]. Russian Journal of Coordination Chemistry 2001. Vol. 27(3), P. 222-226 (In Russ.)].

12. Usacheva T.R., Sharnin V.A., Ledenkov S.F. Thermodynamics of complexation of $\mathrm{Ag}^{+}$with 18-crown-6 in water-dimethyl sulfoxide mixtures. Russian Journal of General Chemistry 2001. Vol. 71(5), P. 707-711.

13. Kuz'mina I.A., Sharnin V.A., Golikov A.N. Complex formation of silver(I) with 18-crown-6 in methanol-DMF binary mixtures. Russian Journal of General Chemistry 2009. Vol. 79(12), P. 25912593.

14. Jabbari A., Hasani M., Shamsipur M. Conductance study of complex formation of thallium and silver ions with several crown ethers in acetonitrile, acetone and dimethylformamide solutions. Journal of inclusion phenomena and molecular recognition in chemistry 1993. Vol. 15(4), P. 329-340.

15. Усачева Т.Р., Леденков С.Ф., Шарнин В.А., Гжейдзяк А. Термодинамика комплексообразования серебра (I) с 18-К-6 в водно-диметилсульфоксидных и водно-этанольных растворителях. Известия вузов. Химия и химическая технология 2000. T. 43(5), C. 87-89. [Usacheva T.R., Ledenkov S.F., Sharnin V.A., Grazac A. Thermodynamics of complexation of silver (I) with 18-K-6 in water-dimethyl sulfoxide and water-ethanol solvents. Russian Journal Izvestia higher educational. Chemistry and chemistry Technology 2000. Vol. 43(5), P. 87-89. (In Russ.)]

16. Golikov A.N., Kuz'mina I.A., Sharnin V.A. Effect of the composition of an acetonitriledimethyl sulfoxide solvent on stability of the silver(I) complexes with 18-crown-6 ether. Russian Journal of Inorganic Chemistry 2007. Vol. 52(11), P. 1739-1740.

17. Volkova M.A., Kuz'mina I.A., Usacheva T.R., Sharnin V.A., Arena G. Effect of the Composition of Ethanol-DMSO Solvents on the Stability of Silver(I) Complexes with 18-Crown-6. Russian Journal of Inorganic Chemistry 2018. Vol. 63(5), P. 687-689.

18. Фиалков В.Я. Растворитель как средство управления химическим процессом. Л.: Химия, 1990. 240 c. [Fialkov V.Ya. Solvent as ameans of controlling the chemical process. Leningrad, Chemistry, 1990. 240 p. (In Russ.)]

19. Кукушкин Ю.Н. Диметилсульфоксид: важнейший апротонный растворитель. Соросовский образовательный журнал 1997. T. 3(9), C. 54-59. [Kukushkin, Yu. N. Dimethyl sulfoxide: the most important aprotic solvent. Soros educational journal 1997. Vol. 3(9), P. $54-59$ (In Russ.)].

20. Измайлов Н.А. Электрохимия растворов. Харьков: Изд-во Харьковского Ордена Трудового Красного Знамени гос ун-та им. А.М. Горького, 1959. 822 с. [Izmailov N.A. Electrochemistry of solutions. Kharkov: Publishing house of the Kharkiv Order of the red banner of Labor State University named after A. M. Gorky, 1959, 822 p. (In Russ.)] 
21. Ковалева М.А. Эффекты среды при образовании гидросульфат-иона и комплекса Ag+ с 18-краун-6-эфиром в растворах. Дис. ... канд. хим. наук. Красноярск, 2014. С. 47. [Kovaleva M.A. The effects of the environment during the formation of the hydrosulfate ion and the complex of Ag + with 18-crown-6-ether in solutions Dissertation for chem. sciences. Krasnoyarsk? 2014, P. 47 (In Russ.)]

22. Оленин С.С., Фадеев Г.Н. Неорганическая химия. М.: Высшая школа, 1979. С. 100 [Olenin S.S., Fadeev G.N. Inorganic chemistry. Moscow: Higher school, 1979. P. 100. (InRuss.)]

23. Ковалева М.А., Феофанов Д.А., Фабинский П.В. Комплексообразование ионов серебра (I) с 18-краун-6-эфиром в смешанных водно-органических растворах. Вестник Таджикского национального университета 2014. T. 1/4, C. 49-53 [Kovaleva M.A., Feofanov D.A., Fabinskii P.V., Complexation of silver (I) ions with 18-crown-6-ether in mixed aqueous organic solutions. Bulletin of the Tajik national University 2014. Vol. 1/4, P. 49-53 (In Russ.)]

24. Пятницкий И.В. Аналитическая химия серебра. М.: Наука, 1975. 261 с. [Pyatnitskiy I.V. Analytical chemistry of silver. Moscow: Science, 1975. 261 p. (In Russ.)]

25. McPartlin M., Masson R. The structure of a bis(dimethylsulphoxide) iridium (III) complex containing a metal-carbon $\sigma$-bond. Chemical Communications (London) 1967. Vol. 11, P. 545-547.

26. BjÖrk N-O., Cassel A. Metal Halide and Pseudohalide Complexes in Dimethyl Sulfoxide Solution. II. The Crystal Structure of Bis dimethyl Sulfoxide Silver (I) Perchlorate. Acta Chemica Scandinavica 1976. Vol. 30A(4), Р. 235-240.

27. Неницеску К.Д. Органическая химия. М.: Изд-во иностр. лит., 1963. 792 с. [Nenitzescu K.D. Organic chemistry. Moscow: Publishing House of foreign literature, 1963. 792 p. (In Russ.)]

28. Васильев В.П., Бородин В.А., Маркова Н.В. Термодинамические характеристики комплексообразования $\mathrm{Ca}^{2+}, \mathrm{Sr}^{2+}$ и $\mathrm{Ba}^{2+}$ с 18-краун-6 в водном растворе. Журнал общей химии 1994. T. 64(11), C. 1910-1913 [Vasiliev V.P., Borodin V.A., Markova N.V. Thermodynamic characteristics of complex formation of $\mathrm{Ca}^{2+}, \mathrm{Sr}^{2+}$ and $\mathrm{Ba}^{2+}$ with 18-crown-6 in aqueous solution. Journal of General chemistry 1994. Vol. 64(11), P. 1910-1913 (In Russ.)] 